examination was normal, and at 6 months in the other patient, hemiparesis was improved. Possible causes for the stroke included varicella angiopathy, small patent foramen ovale, and iron-deficiency anemia. (Wirrell E, Hill MD, Jadavji T, et al. Stroke after varicella vaccination. J Pediatr Dec 2004;145:845-847). (Reprints: Elaine Wirrell MD, FRCPC, Division of Pediatric Neurology, Alberta Children's Hospital, 1820 Richmond Rd SW, Calgary, AB Canada T2T 5C7).

COMMENT. Acute hemiplegia with lacunar stroke has previously been reported after primary varicella infection (Eda I et al. Brain Dev 1983;5:494-499). The above case-reports are the first evidence of cerebral vasculopathy and stroke secondary to varicella vaccine. Patent foramen ovale and iron deficiency anemia were possible predisposing factors. Other neurological complications occurring in temporal association with varicella vaccination have included encephalitis, seizures, neuropathy, and ataxia (American Academy of Pediatrics Red Book 2000;25 thed;633). Acute cerebellar ataxia was reported in a 2 year-old boy who developed vomiting and ataxia 10 days after vaccination. MRI showed multiple demyelinating lesions. Recovery followed in 3 weeks. (Sunaga Y et al. Pediatr Neurol 1995;13:340-342; Ped Neur Briefs Feb 1996). The neurology of the varicella-zoster virus is described in a historical perspective (Nogueira RG, Traynor B. Arch Neurol Dec 2004;61:1974-1977). VZV was first isolated in 1952, using fluorescent-labeled antibodies (Weller T, Stoddard M. J Immunol 68:311-319).

Zoster-associated intracranial hypertension is reported in a 14-year-old girl who presented with headache, vomiting, papilledema, CSF pleocytosis and elevated protein, and herpes zoster with positive VZV DNA in the CSF. The patient had a history of varicella at 4 years of age. Intracranial hypertension is an unusual complication of the reactivation of varicellazoster virus. (Millichap JJ, Freeman JL. Pediatr Neurol 2005;32: in press).

\title{
STREPTOCOCCAL SUBDURAL EMPYEMA AS A COMPLICATION OF VARICELLA
}

A 3-month-old male infant who presented with a group A streptococcal subdural empyema on day 5 of a varicella skin rash is reported from the University of British Columbia, Vancouver, BC, Canada. On day 1 of the rash, he had decreased appetite and vomited twice, on day 2 , fever, and on day 4 , otitis media. On day 5 , he developed rightsided focal seizures with ocular deviation and generalized status epilepticus. CT showed bilateral subdural fluid collections, maximal left. A 5-year-old sibling had developed uncomplicated varicella 2 weeks earlier. On admission to BC Children's Hospital, the varicella rash was diffuse, without secondary bacterial infection. He was irritable, his fontanelle was bulging, and his fundi showed papilledema. Lumbar puncture was not done because of skin lesions and intracranial hypertension. MRI findings were consistent with subdural empyema. A subdural tap yielded $55 \mathrm{ml}$ of purulent fluid with Streptococcus pyogenes, susceptible to penicillin. After 2 weeks treatment with iv. penicillin, focal seizures recurred and he had developed a right-sided hemiparesis with increased size of the left subdural effusion. A subdural empyema was drained at craniotomy. The CSF smears and cultures were negative. Treatment with cefotaxime, vancomycin, and penicillin $G$ was followed by resolution of the subdural empyema, sterile cultures, and slow improvement of 
the hemiparesis. Seizures were controlled with clobazam. The association of GAS subdural empyema and varicella had not previously been described. (Ulloa-Gutierrez R, Dobson S, Forbes J. Group A streptococcal subdural empyema as a complication of varicella. Pediatrics January 2005;115:e112-e114). (Respond: Rolando Ulloa-Gutierrez MD, Pediatric Infectious Diseases Division, British Columbia's Children's Hospital, 4480 Oak St, Rm K4166, Vancouver, BC, Canada V6H 3V4).

COMMENT. Varicella is the most commonly identified risk factor in children with severe invasive group A streptococcal (GAS) infections. The portal of entry is unknown in $50 \%$ of cases, but the skin is the prime suspect. (AAP Red Book, $25^{\text {th }}$ ed;528). GAS bacterial meningitis was presumed to be the source of the subdural empyema in the above case, although the diagnosis was not confirmed by lumbar puncture and CSF examination. Bacteremia and a hematogenous source were other possibilities considered, despite sterile blood cultures. Invasive GAS infections associated with varicella have decreased with vaccination.

\section{VIRAL INFECTIONS AND FEBRILE SEIZURES}

The incidence of febrile seizures (FS) in a cohort of children, ages 3 months to 5 years, living in a Netherlands province was compared with the incidence of common viral infections reported to a national registry and the results reported from the Department of Medical Microbiology, Public Health Laboratory Friesland, Leeuwarden, The Netherlands. In a 4-year period, April 1998 to April 2002, 267 of $303(88 \%)$ general practitioners in the province registered 654 cases of FS (388 first FS and 266 recurrences) in a group of 429 children, an estimated incidence of 2.4 in 1000 patient-years. The ratio of simple versus complex FS was 7.3. A seasonal variation was observed, with peaks in the winter, nadirs during the summer, and small increases at the end of summer. Statistical comparison of the seasonal variation of FS with viral incidence figures showed a significant correlation between FS and influenza A, especially with FS recurrences, and to a lesser degree of significance, with complex FS. Poisson regression analysis revealed no association between FS and RSV infections, despite high rate of infection and clear seasonal variation. Influenza B virus, parainfluenza viruses, enteroviruses, and adenoviruses showed no correlation with FS also, but the incidence of these infections was lower and a seasonal trend could not be demonstrated. Influenza A has a significant role in the recurrence of FS in the Netherlands. Vaccination against influenza should be considered as a preventive therapy for FS recurrence after a first FS. (van Zeijl JH, Mullaart RA, Borm GF, Galama JMD. Recurrence of febrile seizures in the respiratory season is associated with influenza A. J Pediatr December 2004;145:800-805). (Reprints: Dr JH van Zeijl, Public Health Laboratory Friesland, Department of Medical Microbiology, PO Box 21020, 8900 JA Leeuwarden, The Netherlands).

COMMENT. The role of infections in neurologic disease is constantly changing, and epidemiological studies show the emergence of new or the re-emergence of old maladies (Gendelman HE, Persidsky Y. Lancet Neurology January 2005;4:12-13). The association of influenza A with febrile convulsions (FC) has shown changes over time and geographically. In recent correspondence regarding influenza virus and the frequency and mechanism of FC 